\title{
Quantitative versions of the Subspace Theorem and applications
}

\author{
Yann Bugeaud (Strasbourg)
}

\section{Introduction}

The Subspace Theorem, whose name will be clear from its statement, was proved by Wolfgang Schmidt around forty years ago. It provides us with a multidimensional extension of Roth's Theorem and was originally developed for the study of two classical problems, namely algebraic approximation to algebraic numbers and norm form equations (a class of Diophantine equations which includes the Thue equations). Subsequent applications (of suitable extensions of the Subspace Theorem) to unit equations and linear recurrence sequences were published some ten years later, as well as a proof of a conjecture of Lang and many further applications to families of Diophantine equations which include norm form equations.

During the last decade, several quite unexpected applications of the Subspace Theorem were found, some of which have been discussed by Yuri Bilu in his talk at the Séminaire Bourbaki [14]. These include new transcendence criteria, finiteness results for the number of solutions to families of exponential Diophantine equations, and the work of Corvaja and Zannier (with subsequent developments by Autissier and Levin) on integral points on curves and surfaces.

Roughly speaking, the Subspace Theorem asserts that, for every $n \geq 2$, all the integral solutions $\left(x_{1}, \ldots, x_{n}\right)$ to a given system of linear equations with real algebraic coefficients are, under some necessary conditions, contained in a finite union $\mathcal{S}_{1} \cup \ldots \cup \mathcal{S}_{t}$ of proper rational subspaces of $\mathbf{Q}^{n}$. Like Roth's Theorem, it is ineffective, in the sense that its proof does not give an upper bound for the height of the subspaces $\mathcal{S}_{1}, \ldots, \mathcal{S}_{t}$. However, Schmidt was able in 1989 to establish an explicit upper bound for $t$, the number of proper rational subspaces which contain all the solutions. Such a statement is called the Quantitative Subspace Theorem. One of the purposes of this expository text is to show the importance of this quantitative statement and to display some of its (sometimes rather unexpected) consequences.

In Sections 2 and 3 we state the Subspace Theorem and discuss some of its most classical applications. Section 4 is concerned with several recent applications, mainly to transcendence theory. We continue in Section 5 with a statement of a version of the Quantitative Subspace Theorem and mention its applications to equations with unknowns in a finitely generated multiplicative group and to linear recurrence sequences. In Section 6 , we show how a quantitative version of Roth's Theorem can be used to improve, under an extra hypothesis, the conclusion of Roth's Theorem itself in two different directions. We further explain that similar ideas can be worked out with the Quantitative Subspace The- 
orem. Finally, in the last section, we survey several new applications of the Quantitative Subspace Theorem, again mainly to transcendence questions.

We warn the reader that we do not seek for exhaustivity. For instance, we only briefly mention the important works by Evertse, Györy and their coauthors on decomposable form equations and other families of Diophantine equations. As of the recent results, we have chosen not to quote the deep recent works on integral points on curves and surfaces, for which the reader is directed to [14]. Moreover, throughout this text (including the present section), we simply write Subspace Theorem even if we mean an extension of the first version of this theorem. Lastly, the reader is invited to consult the books $[67,70,15$, 74], which are very good references on the subject.

Throughout this text, $\theta$ (resp. $\xi$ ) usually denotes a real number (resp. a real algebraic number).

\section{Roth's Theorem and the Schmidt Subspace Theorem}

It follows from the theory of continued fractions that, for any irrational real number $\theta$, there are infinitely many rational numbers $p / q$ with $q \geq 1$ and

$$
\left|\theta-\frac{p}{q}\right|<\frac{1}{q^{2}}
$$

Let $\varepsilon$ be a positive real number. Since the sum

$$
\sum_{q \geq 1} q \frac{2}{q^{2+\varepsilon}}
$$

converges, the set of real numbers $\theta$ in $(0,1)$ such that

$$
\left|\theta-\frac{p}{q}\right|<\frac{1}{q^{2+\varepsilon}}
$$

holds for infinitely many rational numbers $p / q$ with $q \geq 1$ has zero Lebesgue measure. This can be rephrased by saying that almost all (throughout the present paper, 'almost all' refers to the Lebesgue measure) real numbers are approximable by rational numbers at order 2 and to no better order. In 1955, Roth [59] established that every algebraic irrational number cannot be approximated by rationals at an order greater than 2 and, in this respect, behaves like almost all real numbers.

Theorem (Roth, 1955). Let $\xi$ be an irrational, algebraic real number. Let $\varepsilon$ be a positive real number. Then there are only finitely many rational numbers $p / q$ with $q \geq 1$ such that

$$
\left|\xi-\frac{p}{q}\right|<\frac{1}{q^{2+\varepsilon}}
$$

If we impose additional arithmetical conditions on the numerators and on the denominators of the rational numbers that are good approximations to $\xi$, even a weaker inequality than (2.1) has only finitely many solutions. This was worked out by Ridout [58]. For a prime number $\ell$ and a non-zero rational number $x$, we set $|x|_{\ell}:=\ell^{-u}$, where $u$ is the exponent of $\ell$ in the prime decomposition of $x$. Furthermore, we set $|0|_{\ell}=0$. 
Theorem (Ridout, 1957). Let $S$ be a finite set of prime numbers. Let $\xi$ be an irrational, algebraic real number. Let $\varepsilon$ be a positive real number. The inequality

$$
\prod_{\ell \in S}|p q|_{\ell} \cdot \min \left\{1,\left|\xi-\frac{p}{q}\right|\right\}<\frac{1}{q^{2+\varepsilon}}
$$

has only finitely many solutions in non-zero integers $p, q$.

In particular, it follows from Ridout's Theorem that, for every integer $b \geq 2$, every irrational, algebraic real number $\xi$, every positive integers $s, t$, and every positive $\varepsilon$, the inequality

$$
\left|\xi-\frac{p}{s b^{n}}\right|<\frac{1}{t\left(b^{n}\right)^{1+\varepsilon}}
$$

has only finitely many integer solutions $(p, n)$ with $n \geq 1$.

Roth's Theorem has been subsequently generalized by Wolfgang Schmidt [63, 64, 65, 66]. The first versions of the Schmidt Subspace Theorem that take also non-Archimedean valuations into account were established independently by Dubois and Rhin [32] and Schlickewei $[60,61]$. We quote below a special case of a result from [62].

Theorem (Subspace Theorem). Let $n \geq 2$ be an integer. Let $S$ be a finite set of prime numbers. Let $L_{1, \infty}, \ldots, L_{n, \infty}$ be $n$ linearly independent linear forms in $n$ variables with real algebraic coefficients. For any prime number $\ell$ in $S$, let $L_{1, \ell}, \ldots, L_{n, \ell}$ be $n$ linearly independent linear forms with integer coefficients. Let $\varepsilon$ be a positive real number. Then, the set of solutions $\mathbf{x}=\left(x_{1}, \ldots, x_{n}\right)$ in $\mathbf{Z}^{n}$ to the inequality

$$
\prod_{i=1}^{n}\left|L_{i, \infty}(\mathbf{x})\right| \cdot \prod_{\ell \in S} \prod_{i=1}^{n}\left|L_{i, \ell}(\mathbf{x})\right|_{\ell} \leq\left(\max \left\{1,\left|x_{1}\right|, \ldots,\left|x_{n}\right|\right\}\right)^{-\varepsilon}
$$

lies in finitely many proper subspaces of $\mathbf{Q}^{n}$.

We conclude this section by reformulating Roth's Theorem in order to point out that it is a consequence of the Subspace Theorem.

Theorem (Roth, 1955). Let $\xi$ be a real algebraic number. Let $\varepsilon$ be a positive real number. The integer solutions $(p, q)$ to

$$
q \cdot|q \xi-p|<|q|^{-\varepsilon}
$$

are contained in a finite union of proper subspaces of $\mathbf{Q}^{2}$.

The above formulation of Roth's Theorem asserts that there is a finite set of equations $x_{1} X+y_{1} Y=0, \ldots, x_{t} X+y_{t} Y=0$ such that, for every solution $(p, q)$ to $(2.3)$, there exists an integer $k=1, \ldots, t$ with $x_{k} p+y_{k} q=0$. This means that, if $\xi$ is irrational, then there are only finitely many rational solutions to $|\xi-p / q|<|q|^{-2-\varepsilon}$. 


\section{Some classical applications of the Subspace Theorem}

We begin with the extension of Roth's Theorem to the approximation to real algebraic numbers by real algebraic numbers of bounded degree established in [65]. Throughout this text, the height $H(\xi)$ of an algebraic number $\xi$ is the maximum of the absolute values of the coefficients of its minimal defining polynomial over the integers.

Theorem (Schmidt, 1970). Let $n$ be a positive integer. Let $\xi$ be a real algebraic number of degree greater than $n$. Let $\varepsilon$ be a positive real number. Then, there exist only finitely many algebraic numbers $\alpha$ of degree at most $n$ such that

$$
|\xi-\alpha|<H(\alpha)^{-n-1-\varepsilon}
$$

Furthermore, there exist infinitely many algebraic numbers $\alpha$ of degree at most $n$ such that

$$
|\xi-\alpha|<H(\alpha)^{-n-1+\varepsilon} .
$$

The former assertion of the above theorem is a direct application of the Subspace Theorem, while the latter one rests also on a classical transference principle. As far as approximation by real algebraic numbers of degree at most $n$ is concerned, real algebraic numbers of degree exceeding $n$ behave like almost all real numbers. However, and surprisingly, for every even positive integer $n$, there exist complex non-real algebraic numbers of degree $n+2$ which are much better approximable by complex algebraic numbers of degree $n$ than are almost all complex numbers. See [22] for a study of the approximation to complex algebraic numbers by algebraic numbers of bounded degree. This problem remains, at the moment, not completely solved.

We continue by a deep extension of Thue's finiteness result to a more general class of equations. Let $L\left(X_{1}, \ldots, X_{n}\right)=\alpha_{1} X_{1}+\ldots+\alpha_{n} X_{n}$ be a linear form with coefficients in a number field K. By means of his Subspace Theorem, Schmidt [66] established a necessary and sufficient condition on the norm form $N_{\mathbf{K} / \mathbf{Q}}\left(L\left(X_{1}, \ldots, X_{n}\right)\right)$ that ensures that the norm form equation

$$
N_{\mathbf{K} / \mathbf{Q}}\left(L\left(x_{1}, \ldots, x_{n}\right)\right)=m, \quad\left(x_{1}, \ldots, x_{n}\right) \in \mathbf{Z}^{n},
$$

has only finitely many solutions for every non-zero integer $m$.

Recall that if $S$ is a finite set of valuations (on a given number field $\mathbf{K}$ ) which contains all the infinite valuations, then an $S$-unit $x$ in $\mathbf{K}$ is characterised by $|x|_{v}=1$ for all $v \notin S$. We continue this section with an important result on $S$-unit equations from [57, 33]. We point out that numerous Diophantine problems can be reduced to equations belonging to this class.

Theorem (Schlickewei and van der Poorten, 1982, and Evertse, 1984). Let K be a number field and $S$ a finite set of places of $\mathbf{K}$ containing all Archimedean places. Let $q \geq 2$ be an integer. Let $\chi$ be the set of solutions to

$$
x_{1}+\ldots+x_{q}=1
$$


such that $x_{1}, \ldots, x_{q}$ are $S$-units in $\mathbf{K}$ and no proper subsum of $x_{1}+\ldots+x_{q}$ vanishes. Then, $\chi$ is a finite set.

We conclude this section with a very brief overview of subsequent development between 1982 and 1998. In 1984, Laurent [51] applied the Subspace Theorem to establish a conjecture of Lang. A few years later, Evertse and Győry [38] extended Schmidt's results on norm form equations and proved finiteness criteria for decomposable form equations (which is a class of Diophantine equations which includes norm form equations, discriminant form equations and index form equations).

Evertse, Győry, Stewart, and Tijdeman [40, 41] established by means of the Subspace Theorem that most of the $S$-unit equations in two unknowns have at most two solutions. More precisely, let $\mathbf{K}$ be a number field and $S$ a finite set of valuations on $\mathbf{K}$ containing all the infinite valuations. Let $a_{1}, a_{2}, a_{3}$ be non-zero elements in $\mathbf{K}$ and consider the equation $a_{1} x+a_{2} y=a_{3}$ in the unknown $S$-units $x, y$ in $\mathbf{K}$. Two such equations are said to be $S$-equivalent if their coefficient sets become equal after a possible permutation and multiplication by $S$-units. In [40] the authors prove that there is a finite set of classes such that any equation not belonging to it has at most two solutions.

\section{Some more recent applications of the Subspace Theorem}

Many new applications of the Subspace Theorem were found by Corvaja and Zannier, in a series of papers starting in 1998 with [25]. We content ourselves to quote four of their numerous results.

Let $\mathbf{K}$ be a field included in the set of complex numbers. We call a $\mathbf{K}$-power sum any expression of the form

$$
u(n)=b_{1} a_{1}^{n}+\ldots+b_{m} a_{m}^{n},
$$

where $a_{1}, \ldots, a_{m}$ are distinct non-zero elements of $\mathbf{K}$ (called the roots) and $b_{1}, \ldots, b_{m}$ are in $\mathbf{K}$. We begin with a result from [26].

Theorem (Corvaja and Zannier, 2002). Let $u$ be a Q-power sum with positive roots and $P(X, Y) \in \mathbf{Q}[X, Y]$ be a polynomial non-constant in $Y$. Assume that the equation $P(u(n), y)=0$ has infinitely many solutions in integers $n$ and $y$. Then, there exists a $\overline{\mathbf{Q}}-$ power sum $v$ with positive real coefficients such that $P(u(n), v(n))=0$ for all integers $n$.

We continue with an arithmetical application of the Subspace Theorem extracted from $[20]$.

Theorem (Bugeaud, Corvaja and Zannier, 2003). Let $a$ and $b$ be coprime integers with $a>b \geq 2$. For any positive real number $\varepsilon$, there exists an integer $n_{0}(\varepsilon)$ such that

$$
\operatorname{gcd}\left(a^{n}-1, b^{n}-1\right)<2^{\varepsilon n}, \quad \text { for every } n \geq n_{0}(\varepsilon) .
$$

This theorem was subsequently generalized in various directions, and its extension [27] implies a positive answer to a conjecture of Győry, Sárközy and Stewart [48] asserting that if $a, b, c$ are positive integers with $a>b>c$, then $P[(a b+1)(b c+1)(c a+1)]$ tends to 
infinity as $a$ tends to infinity, where $P[x]$ denotes the greatest prime factor of the integer $x \geq 2$.

Theorem (Corvaja and Zannier, 2003). If $a, b, c$ are positive integers with $a>b>c$, then

$$
P[(a b+1)(a c+1)] \longrightarrow \infty
$$

as a tends to infinity.

Independently, Hernández and Luca [49] established the conjecture of Győry, Sárközy and Stewart [48], but not under the strong form in the above theorem.

We end this section with several recent results on transcendence. A first one, established in [26], asserts that lacunary series take transcendental values at algebraic arguments.

Theorem (Corvaja and Zannier, 2002). Let $\left(n_{j}\right)_{j \geq 1}$ be a strictly increasing sequence of positive integers satisfying

$$
\liminf _{j \rightarrow+\infty} \frac{n_{j+1}}{n_{j}}>1
$$

Then, the function $f$ defined for $z$ in the open unit disc by

$$
z \mapsto f(z)=\sum_{j \geq 1} z^{n_{j}}
$$

takes transcendental values at every non-zero algebraic point in the open unit disc.

A second result is a combinatorial transcendence criterion derived from the Subspace Theorem and established in [8]. Let $b \geq 2$ be an integer. For a non-zero real number $\theta$, write

$$
\theta= \pm \sum_{k \geq-k_{0}} \frac{a_{k}}{b^{k}}=a_{-k_{0}} \ldots a_{0} \cdot a_{1} a_{2} \ldots
$$

where $k_{0} \geq 0, a_{-k_{0}} \neq 0$ if $k_{0}>0$, the $a_{k}$ 's are integers from $\{0,1, \ldots, b-1\}$ and $a_{k}$ is different from $b-1$ for infinitely many indices $k$. The sequence $\left(a_{k}\right)_{k \geq-k_{0}}$ is uniquely determined by $\theta$ : it is its $b$-ary expansion.

Theorem (Adamczewski, Bugeaud and Luca, 2004). Keep the above notation. If there exist a positive real number $\varepsilon$ and infinitely many triples of positive integers $(j, k, \ell)$ such that

$$
a_{j+i}=a_{j+k+i}, \quad i=1, \ldots, \ell
$$

and

$$
\ell \geq \varepsilon(j+k), \quad \ell \leq k
$$

then $\theta$ is either rational, or transcendental.

The assumption of the above theorem means that there are blocks of arbitrarily large length $\ell$ that occur twice very near to the beginning of the $b$-ary expansion of $\theta$. 
Proof. Assume for simplicity that $0<\theta<1$. Let $\left(j_{1}, k_{1}, \ell_{1}\right),\left(j_{2}, k_{2}, \ell_{2}\right), \ldots$ be an infinite sequence of triples of positive integers satisfying (4.2), (4.3) and $k_{1}<k_{2}<\ldots$ Let $m$ be a positive integer. Define the rational number

$$
\theta_{m}=0 \cdot a_{1} a_{2} \ldots a_{j_{m}} a_{j_{m}+1} \ldots a_{j_{m}+k_{m}} a_{j_{m}+1} \ldots a_{j_{m}+k_{m}} \ldots a_{j_{m}+1} \ldots a_{j_{m}+k_{m}} \ldots
$$

with preperiod $a_{1} a_{2} \ldots a_{j_{m}}$ and period $a_{j_{m}+1} \ldots a_{j_{m}+k_{m}}$. There exists an integer $p_{m}$ such that

$$
\theta_{m}=\frac{p_{m}}{b^{j_{m}}\left(b^{k_{m}}-1\right)}
$$

Furthermore, since, by (4.2),

$$
\theta=0 \cdot a_{1} a_{2} \ldots a_{j_{m}} a_{j_{m}+1} \ldots a_{j_{m}+k_{m}} a_{j_{m}+1} \ldots a_{j_{m}+\ell_{m}} \ldots
$$

the real numbers $\theta_{m}$ and $\theta$ have (at least) their first $j_{m}+k_{m}+\ell_{m}$ digits in common, and

$$
\left|\theta-\theta_{m}\right|=\left|\theta-\frac{p_{m}}{b^{j_{m}}\left(b^{k_{m}}-1\right)}\right|<\frac{1}{b^{j_{m}+k_{m}+\ell_{m}}}
$$

Multiplying by the denominator and using (4.3), this gives

$$
\left|b^{j_{m}+k_{m}} \theta-b^{j_{m}} \theta-p_{m}\right|<\left(b^{j_{m}+k_{m}}\right)^{-\ell_{m} /\left(j_{m}+k_{m}\right)} \leq\left(b^{j_{m}+k_{m}}\right)^{-\varepsilon} .
$$

Consider the following three linearly independent linear forms with real algebraic coefficients:

$$
\begin{aligned}
& L_{1, \infty}\left(X_{1}, X_{2}, X_{3}\right)=X_{1}, \\
& L_{2, \infty}\left(X_{1}, X_{2}, X_{3}\right)=X_{2}, \\
& L_{3, \infty}\left(X_{1}, X_{2}, X_{3}\right)=\theta X_{1}-\theta X_{2}-X_{3} .
\end{aligned}
$$

Let $S$ be the set of prime divisors of $b$. For any prime number $\ell$ in $S$, consider the following three linearly independent linear forms with integer coefficients:

$$
\begin{aligned}
& L_{1, \ell}\left(X_{1}, X_{2}, X_{3}\right)=X_{1}, \\
& L_{2, \ell}\left(X_{1}, X_{2}, X_{3}\right)=X_{2}, \\
& L_{3, \ell}\left(X_{1}, X_{2}, X_{3}\right)=X_{3} .
\end{aligned}
$$

We deduce from (4.4) that

$$
\prod_{i=1}^{3}\left|L_{i, \infty}\left(\left(b^{j_{m}+k_{m}}, b^{j_{m}}, p_{m}\right)\right)\right| \cdot \prod_{\ell \in S} \prod_{i=1}^{3}\left|L_{i, \ell}\left(\left(b^{j_{m}+k_{m}}, b^{j_{m}}, p_{m}\right)\right)\right|_{\ell} \leq\left(b^{j_{m}+k_{m}}\right)^{-\varepsilon} .
$$

Assume that $\theta$ is algebraic. It then follows from the Subspace Theorem that the set of triples $\left(b^{j_{m}+k_{m}}, b^{j_{m}}, p_{m}\right), m \geq 1$, is contained in a finite union of rational subspaces of 
$\mathbf{Q}^{3}$. Consequently, there exist a non-zero triple $\left(z_{1}, z_{2}, z_{3}\right)$ of integers and an infinite set $\mathcal{M}$ of positive integers such that

$$
z_{1} b^{j_{m}+k_{m}}+z_{2} b^{j_{m}}+z_{3} p_{m}=0
$$

for $m$ in $\mathcal{M}$. If $\left(k_{m}\right)_{m \in \mathcal{M}}$ is bounded, then there is a positive integer $k$ and arbitrarily large integers $m$ such that

$$
\left|\theta-\theta_{m}\right|=\left|\theta-\frac{p_{m}}{b^{j_{m}}\left(b^{k}-1\right)}\right|<\frac{1}{b^{k}\left(b^{j_{m}}\right)^{1+\varepsilon}},
$$

and it follows from Ridout's Theorem (see (2.2)) that $\theta$ must be rational. Thus, we may assume that $k_{1}<k_{2}<\ldots$ Dividing both sides of (4.5) by $b^{j_{m}}\left(b^{k_{m}}-1\right)$ and letting $m$ tend to infinity, we conclude that $\theta$ is rational. This completes the proof of the transcendence criterion.

A similar application of the Subspace Theorem was used previously by Troi and Zannier [73] to prove the transcendence of the number $\alpha=\sum_{a \in S} 2^{-a}$, where $S$ denotes the set of integers which can be represented as sums of distinct terms of the form $2^{k}+1$ with $k$ being a positive integer. Also, Mahler [54] suggested explicitly to apply the Subspace Theorem exactly as in the above proof to investigate whether the middle third Cantor set contains or not irrational algebraic elements! Other applications of the Subspace Theorem to transcendence can be found in $[63,56,24]$.

Very little is known about the expansion of an irrational algebraic number to an integer base. It is, however, believed that every algebraic irrational $\xi$ shares most of the properties of almost all numbers. In particular, its $b$-ary expansion is likely to be normal. This means that, for every positive $n$, each combination of $n$ digits from $\{0,1, \ldots, b-1\}$ is likely to occur with the same frequency, namely $1 / b^{n}$, in the $b$-ary expansion of $\xi$.

One of the many ways to measure the complexity of a real number is to use the block complexity. For a real number $\theta$, an integer $b \geq 2$, and a positive integer $n$, denote by $p(n, \theta, b)$ the total number of distinct blocks of $n$ digits in the $b$-ary expansion of $\theta$, that is,

$$
p(n, \theta, b)=\operatorname{Card}\left\{\left(a_{h+1}, \ldots, a_{h+n}\right): h \geq 0\right\} .
$$

Obviously, we have

$$
1 \leq p(n, \theta, b) \leq b^{n}, \quad \text { for } n \geq 1 .
$$

It is shown in [2] with the help of the above combinatorial transcendence criterion that the complexity function $n \mapsto p(n, \xi, b)$ of an irrational algebraic number $\xi$ grows faster than any linear function. A weaker result from $[44,10]$, which follows from a weaker transcendence criterion (namely Ridout's Theorem), asserts that $p(n, \xi, b)-n$ tends to infinity with $n$.

Theorem (Adamczewski and Bugeaud, 2007). If $\xi$ is an algebraic irrational real number, then

$$
\lim _{n \rightarrow \infty} \frac{p(n, \xi, b)}{n}=+\infty
$$


The key combinatorial (and easy) lemma for this proof asserts that if for a sequence $\left(a_{h}\right)_{h \geq 1}$ of elements from $\{0,1, \ldots, b-1\}$ there exist a positive $C$ and arbitrarily large integers $n$ such that

$$
\operatorname{Card}\left\{\left(a_{h+1}, \ldots, a_{h+n}\right): h \geq 0\right\} \leq C n,
$$

then there are a positive real number $\varepsilon$ and infinitely many triples of positive integers $(j, k, \ell)$ with $(4.2)$ and (4.3).

Similar ideas yield new examples of transcendental continued fractions. The next statement is extracted from [1].

Theorem (Adamczewski and Bugeaud, 2005). Let $\left(d_{j}\right)_{j \geq 1}$ be a bounded sequence of positive integers such that there exist a positive real number $\varepsilon$ and arbitrarily large integers $\ell$ such that

$$
d_{\ell+i}=d_{i}, \quad \text { for all integers } i \text { with } 1 \leq i \leq \varepsilon \ell .
$$

Then, the real number $\left[0 ; d_{1}, d_{2}, \ldots\right]$ is either quadratic, or transcendental.

Further results can be found in $[3,4,7]$. Recall that we still do not know whether there exist algebraic numbers with an unbounded sequence of partial quotients in their continued fraction expansion, nor whether there exist algebraic numbers of degree at least three with a bounded sequence of partial quotients.

\section{Quantitative statements and some of their applications}

Unfortunately, the proof of Roth's Theorem does not enable us to get explicit upper bounds for the height of the solutions to (2.1). Nevertheless, it can be used to derive an explicit upper bound for the number of reduced solutions to (2.1). This was first done in 1955 by Davenport and Roth [31].

Theorem DR (Davenport and Roth, 1955). Let $\xi$ be an algebraic real number of degree $d$ and height at most $H$. Let $\varepsilon$ be a positive real number with $\varepsilon \leq 1 / 3$. Then there are no more than

$$
2(2 H)^{2 / \varepsilon}+4 \varepsilon^{-1} \log \log \left(1+2 H^{d}\right)+\exp \left\{285 d^{2} \varepsilon^{-2}\right\}
$$

reduced rational numbers $p / q$ with $q \geq 1$ such that

$$
0<\left|\xi-\frac{p}{q}\right|<\frac{1}{q^{2+\varepsilon}}
$$

The above theorem was subsequently improved by several authors and the best known result is established in the Appendix of [21].

The Subspace Theorem suffers from the same lack of effectivity as Roth's Theorem; nonetheless, Schmidt [68] was able to give explicit bounds for the number of exceptional subspaces. To avoid a too technical statement, we quote a theorem of Schmidt with the first result of this type. The height of a linear form $L=\alpha_{1} x_{1}+\ldots+\alpha_{n} x_{n}$ with algebraic coefficients is denoted by $H(L)$ and satisfies $H(L) \leq n^{d / 2}(d+1)^{n / 2} H\left(\alpha_{1}\right) \times \ldots \times H\left(\alpha_{n}\right)$, where $d$ is the degree of a number field containing $\alpha_{1}, \ldots, \alpha_{n}$. 
Theorem (Schmidt, 1989). Let $n \geq 2$ be an integer. Let $L_{1}, \ldots, L_{n}$ be linearly independent linear forms in $n$ variables with coefficients in a real algebraic number field of degree $d$. Let $\varepsilon$ be a real number satisfying $0<\varepsilon<1$. Then there are proper rational subspaces $\mathcal{S}_{1}, \ldots, \mathcal{S}_{t}$ of $\mathbf{Q}^{n}$, where

$$
t=\left[(2 d)^{2^{26 n} \varepsilon^{-2}}\right],
$$

such that all integer solutions $\mathbf{x}=\left(x_{1}, \ldots, x_{n}\right)$ to

$$
\left|L_{1}(\mathbf{x}) \cdots L_{n}(\mathbf{x})\right|<\left|\operatorname{det}\left(L_{1}, \ldots, L_{n}\right)\right| \cdot\left(\max \left\{1,\left|x_{1}\right|, \ldots,\left|x_{n}\right|\right\}\right)^{-\varepsilon}
$$

lie in the union of $\mathcal{S}_{1}, \ldots, \mathcal{S}_{t}$ and of the ball

$$
\max \left\{\left|x_{1}\right|, \ldots,\left|x_{n}\right|\right\} \leq \max \left\{(n !)^{8 / \varepsilon}, H\left(L_{1}\right), \ldots, H\left(L_{n}\right)\right\} .
$$

A more general result, including non-Archimedean places, has been established by Schlickewei and improved by Evertse and Schlickewei [42]. A further improvement has been obtained by Evertse and Ferretti [37]; see the expository paper by Evertse [36].

As a first application of his Quantitative Subspace Theorem, Schmidt [69] derived an explicit, uniform bound for the number of solutions to norm-form equations (3.1) depending only on $n, m$ and the degree of the field $\mathbf{K}$. His result was subsequently generalized to decomposable form equations by Győry [45, 46] and Evertse and Győry [39]. Using results from [46], Győry [47] established a very nice theorem on irreducibility of neighbouring polynomials; namely, if $P(X)$ is an integer polynomial, then there exists an effectively computable number $c$, depending only on the degree of $P(X)$ and on its leading coefficient, and a rational integer $b$ of absolute value at most $c$ for which the polynomial $P(X)+b$ is irreducible over the rationals. Perhaps, the same statement holds with a constant $c$ depending only on the degree of $P(X)$, this is an interesting open problem.

A further application of the Quantitative Subspace Theorem is concerned with $S$-unit equations. We quote a more general result, established in [43].

Theorem (Evertse, Schlickewei and Schmidt, 2002). Let $a_{0}, \ldots, a_{q}$ be complex numbers. Let $\Gamma$ be a multiplicative subgroup of $\left(\mathbf{C}^{\times}\right)^{q}$ of rank $r$. Then, the equation

$$
a_{0} x_{0}+\ldots+a_{q} x_{q}=0
$$

in projective points $\left(x_{1}, \ldots, x_{q}\right)$ with coordinates in $\Gamma$ has at most

$$
\exp \left\{(6 q)^{3 q}(r+1)\right\}
$$

solutions where no proper, nonempty subsum of $a_{0} x_{0}+\ldots+a_{q} x_{q}$ vanishes.

The bound (5.1) has been very recently reduced to

$$
(8 q)^{4 q^{4}(q+r+1)}
$$

by Amoroso and Viada [11]. Their improvement comes from a better estimate for the number of solutions of 'very small height', a step of the proof which is independent of the Quantitative Subspace Theorem.

To conclude this section, we quote a striking result on linear recurring sequences [71]. 
Theorem (Schmidt, 2000). Let $u$ be a linear recurrence sequence of order $t$ of complex numbers, which may be degenerated. Then the set of subscripts $k$ with $u_{k}=0$ is the union of at most

$$
\exp \exp \exp (20 t)
$$

arithmetic progressions and single numbers.

We emphasize that the upper bound (5.2) depends only on the order of the recurrence. It has been reduced to

$$
\exp \exp \left(t^{\sqrt{11 t}}\right)
$$

by Allen [9], using a better bound in an auxiliary lemma dealing with the linear independence of certain vectors. A subsequent sharpening can be found in [12].

\section{Two old results as consequences of the quantitative statements}

Roth's Theorem (1955) has a long history. Liouville established in 1844 that a non-zero real algebraic number cannot be approximated by rational numbers at an order greater than its degree. His result was subsequently improved by Thue (1909), Siegel (1921) and later by Dyson (1947) and Gelfond (1948). In the meantime, in 1936, Schneider [72] proved an important, and almost forgotten, result.

Theorem (Schneider, 1936). Let $\xi$ be an irrational, algebraic real number. Let $\varepsilon$ be a positive real number. Let $\left(p_{j} / q_{j}\right)_{j \geq 1}$ be the sequence of reduced rational solutions of

$$
\left|\xi-\frac{p}{q}\right|<\frac{1}{q^{2+\varepsilon}}
$$

ordered such that $3 \leq q_{1}<q_{2}<\ldots$ Then either the sequence $\left(p_{j} / q_{j}\right)_{j \geq 1}$ is finite, or

$$
\limsup _{j \rightarrow+\infty} \frac{\log q_{j+1}}{\log q_{j}}=+\infty
$$

Looking at the theorem of Schneider, let us rewrite Roth's Theorem under the following form.

Theorem (Roth, 1955). Let $\theta$ be an irrational real number. Let $\varepsilon$ be a positive real number. If there is an infinite sequence $\left(p_{j} / q_{j}\right)_{j \geq 1}$ of reduced rational solutions of

$$
\left|\theta-\frac{p}{q}\right|<\frac{1}{q^{2+\varepsilon}}
$$

then $\theta$ is transcendental.

It is natural to ask what kind of results could be obtained if we add in Roth's Theorem an assumption on the denseness of the infinite sequence of solutions, like in Schneider's Theorem. 
We may hope to replace in Schneider's Theorem $\varepsilon$ by a function $q \mapsto \varepsilon(q)$ tending to zero as $q$ increases to infinity. We may also hope to improve the conclusion of Roth's Theorem, that is, to say something more than the mere fact that $\xi$ must be transcendental, for instance, to give transcendence measures for $\xi$.

This is exactly what happens! We first quote a result found by Cugiani [28] in 1958, that improves Schneider's Theorem.

Theorem (Cugiani, 1958). Let $\xi$ be a real algebraic number of degree $d$. For an integer $q \geq 16$, set

$$
\varepsilon(q)=\frac{9 d}{(\log \log \log q)^{1 / 2}} .
$$

Let $\left(p_{j} / q_{j}\right)_{j \geq 1}$ be the sequence of reduced rational solutions of

$$
\left|\xi-\frac{p}{q}\right|<\frac{1}{q^{2+\varepsilon(q)}}
$$

ordered such that $16 \leq q_{1}<q_{2}<\ldots$ Then either the sequence $\left(p_{j} / q_{j}\right)_{j \geq 1}$ is finite, or

$$
\limsup _{j \rightarrow+\infty} \frac{\log q_{j+1}}{\log q_{j}}=+\infty
$$

The above theorem was subsequently generalized by Cugiani [29, 30] and Mahler [53] to include non-Archimedean valuations, and is now referred to as the Cugiani-Mahler Theorem.

The other strengthening of Schneider's Theorem evoked above was worked out by Alan Baker [13]. Following Koksma [50], for a real number $\theta$ and a positive integer $d$, we denote by $w_{d}^{*}(\theta)$ the supremum of the real numbers $w^{*}$ for which the inequality

$$
0<|\theta-\alpha|<H(\alpha)^{-w^{*}-1}
$$

has infinitely many solutions in algebraic numbers $\alpha$ of degree at most $d$. By means of the exponents $w_{d}^{*}$, Koksma divided the set of transcendental numbers into three disjoint classes $S^{*}, T^{*}$ and $U^{*}$. He proved that these classes coincide, respectively, with the classes $S, T$ and $U$ defined by Mahler [52]. A $U^{*}$-number is precisely a transcendental real number $\theta$ for which there exist a positive integer $d$ and, for every positive real number $w$, an algebraic number $\alpha$ of degree $d$ such that $|\theta-\alpha|<H(\alpha)^{-w}$. Liouville numbers are $U^{*}$-numbers. See Chapter 3 from [16] for more information.

Theorem (Baker, 1964). Let $\theta$ be an irrational real number. Let $\varepsilon$ be a positive real number. Assume that there is an infinite sequence $\left(p_{j} / q_{j}\right)_{j \geq 1}$ of reduced rational solutions to

$$
\left|\theta-\frac{p}{q}\right|<\frac{1}{q^{2+\varepsilon}}
$$

that satisfies

$$
\limsup _{j \rightarrow+\infty} \frac{\log q_{j+1}}{\log q_{j}}<+\infty
$$


Then, there exists a constant $\kappa$, depending only on $\theta$ and on $\varepsilon$, such that

$$
w_{d}^{*}(\theta) \leq \exp \exp \kappa d^{2}
$$

for every integer $d \geq 1$. Consequently, $\theta$ is either an $S$-number, or a $T$-number.

The original proofs of the Cugiani-Mahler Theorem and of the Baker Theorem are quite intricate: the authors performed some suitable modifications in the heart of the proof of Roth's Theorem.

It was shown recently (resp. in [17] and in [5]) that both the theorems of CugianiMahler and of Baker can be easily deduced from a quantitative version of Roth's Theorem. Of course, one cannot claim that the resulting new proofs are easier than the original ones, since the difficulties are hidden in the proof of a quantitative version of Roth's Theorem. Nevertheless, keeping in mind that we have a quantitative version of the Subspace Theorem at our disposal, they provide us with a natural way to establish multidimensional extensions of the theorems of Cugiani-Mahler and of Baker, worked out in [17] and in [5], respectively.

We show below how to derive a version of the Cugiani-Mahler Theorem from a quantitative version of Roth's Theorem.

Deduction of a slightly weaker version of the Cugiani-Mahler Theorem from the theorem of Davenport and Roth.

Let $\eta: \mathbf{Z}_{\geq 1} \rightarrow \mathbf{R}_{>0}$ be a non-increasing function. Let $\left(p_{j} / q_{j}\right)_{j \geq 1}$ be the sequence of reduced rational solutions of

$$
\left|\xi-\frac{p}{q}\right|<\frac{1}{q^{2+\eta(q)}}
$$

ordered such that $16 \leq q_{1}<q_{2}<\ldots$ Assume that this sequence is infinite and that there exists an integer $c \geq 2$ such that $q_{j+1} \leq q_{j}^{c}$ for $j \geq 1$.

Let $T$ be a positive integer and set $\eta=\eta\left(q_{T}\right)$. Since $\eta$ is non-increasing, there are at least $T$ reduced rational solutions $p / q$ to

$$
\left|\xi-\frac{p}{q}\right|<\frac{1}{q^{2+\eta}}
$$

Furthermore, our assumption implies that

$$
q_{T} \leq q_{1}^{c^{T}}
$$

We infer from the theorem of Davenport and Roth that

$$
T \leq \exp \left\{c(\xi) \eta^{-2}\right\}
$$

for some positive real number $c(\xi)$ depending only on $\xi$. With the choice

$$
\eta(q)=(\log \log \log q)^{-1 / 2+\delta},
$$

where $\delta$ is an arbitrary positive real number, one obtains

$$
T \leq \exp \left\{c(\xi)\left(\log T+\log \log c q_{1}\right)^{1-\delta}\right\},
$$


and we have reached a contradiction when $T$ is sufficiently large. We have thus obtained a slightly weaker form of Cugiani's Theorem, with a function $\eta$ that decreases slightly slower than the function $\varepsilon$ defined in (6.1).

Following this approach, the use of Evertse's estimate [35] in place of the theorem of Davenport and Roth allows us [17] to show that Cugiani's Theorem remains true with the funtion $\varepsilon$ given by

$$
\varepsilon(q)=\frac{\log \log \log q}{(\log \log q)^{1 / 3}}, \quad \text { for } q \geq 16 .
$$

An even better result (with, essentially, the exponent $1 / 3$ in (6.3) replaced by $1 / 2$ ) follows from $[36,37]$.

By means of the Quantitative Subspace Theorem, it is possible to extend Cugiani's Theorem to approximation by algebraic numbers of bounded degree and to do a first step towards a (small) improvement on the result of Schmidt quoted at the beginning of Section 3.

Theorem (Bugeaud, 2007). Let $n$ be a positive integer and $\xi$ be a real algebraic number of degree greater than $n$. Let $\varepsilon: \mathbf{Z}_{\geq 1} \rightarrow \mathbf{R}_{>0}$ be a non-increasing function satisfying

$$
\lim _{H \rightarrow+\infty} \frac{\varepsilon(H)}{(\log \log H)^{-1 /(2 n+6)}}=+\infty
$$

Let $\left(\alpha_{j}\right)_{j \geq 1}$ be the sequence of distinct algebraic numbers of degree at most $n$ that satisfy

$$
|\xi-\alpha|<H(\alpha)^{-n-1-\varepsilon(H(\alpha))}
$$

ordered such that $1 \leq H\left(\alpha_{1}\right) \leq H\left(\alpha_{2}\right) \leq \ldots$ If this sequence is infinite, then

$$
\limsup _{j \rightarrow+\infty} \frac{\log H\left(\alpha_{j+1}\right)}{\log H\left(\alpha_{j}\right)}=+\infty
$$

Applying the new result of Evertse and Ferretti [37] in place of [42], it is possible to replace the exponent $1 /(2 n+6)$ in $(6.4)$ by an absolute constant.

We conclude this section by a sketch of a new proof of Baker's Theorem. It rests on an apparently slight, nevertheless important, improvement of the theorem of Davenport and Roth that was obtained in 1974 by Mignotte [55].

Theorem (Mignotte, 1974). Let $\xi$ be an algebraic real number of degree $d$ and of height at most $H$, with $H \geq 2$. Let $\varepsilon$ be a positive real number with $\varepsilon<0.01$. Then there exist positive constants

$$
c_{1}(d, \varepsilon)=\exp \left\{2 \varepsilon^{-2}(\log 2 d)\right\}
$$

and

$$
c_{2}(d, \varepsilon)=\exp \left\{\exp \left\{100 \varepsilon^{-2}(\log 2 d)\right\}\right\}
$$


such that there are no more than $c_{1}(d, \varepsilon)$ reduced rational numbers $p / q$ with $q \geq H^{c_{2}(d, \varepsilon)}$ and

$$
0<\left|\xi-\frac{p}{q}\right|<\frac{1}{q^{2+\varepsilon}}
$$

Mignotte's result asserts that the number of solutions to (2.1) whose denominator exceeds some function of $\varepsilon$, the height of $\xi$ and its degree is bounded from above only in terms of $\varepsilon$ and the degree of $\xi$. Actually, this also follows from the proof of the theorem of Davenport and Roth, but these authors did not make the distinction between 'small' and 'large' solutions. Otherwise, they would have established a weaker version of the theorem of Mignotte in which the factor ( $\log 2 d)$ occurring in (6.5) and (6.6) would have been replaced at both places by $d^{2}$ times some numerical constant. The improved dependence on $d$ in the theorem of Mignotte is due to the use of a refined auxiliary lemma in the heart of the proof of Roth's Theorem, see Appendix A from [53].

Deduction of an improved version of the theorem of Baker from the theorem of Mignotte.

By (6.2), there exists a positive real number $c$ such that

$$
q_{j+1} \leq q_{j}^{c}, \quad \text { for } j \geq 1
$$

We begin by showing that $\theta$ is not a Liouville number. To this end, observe that $p_{j} / q_{j}$ and $p_{j+1} / q_{j+1}$ are convergents to $\theta$ with $q_{j+1}>q_{j}$. Since the theory of continued fractions implies that any convergent $p / q$ to $\theta$ with $q_{j} \leq q<q_{j+1}$ satisfies

$$
\left|\theta-\frac{p}{q}\right|>\frac{1}{2 q q_{j+1}} \geq \frac{1}{2 q^{1+c}},
$$

we get the upper bound $w_{1}^{*}(\theta) \leq c$. Hence, $\theta$ is not a Liouville number.

Assume that $\varepsilon<0.01$ and that $q_{1}$ is sufficiently large in order that

$$
\left|\theta-\frac{p_{j}}{q_{j}}\right|<\frac{1}{2 q_{j}^{2+\varepsilon / 2}}, \quad(j \geq 1) .
$$

Let $d$ be an integer, $d \geq 2$, and let $\xi$ be an algebraic real number of degree $d$ and height $H(\xi) \geq 2$. Let $j$ be such that

$$
q_{j-1} \leq H(\xi)^{c_{2}(d, \varepsilon / 2)}<q_{j}
$$

where $c_{2}(d, \varepsilon / 2)=\exp \left\{\exp \left\{400 \varepsilon^{-2}(\log 2 d)\right\}\right.$.

Define the real number $\chi$ by

$$
|\theta-\xi|=H(\xi)^{-\chi}
$$

We will bound $\chi$ from above in terms of $d$. 
Set $v=2+\varepsilon / 2$. Let $T$ be the greatest integer for which $2 q_{j}^{v c^{T-1}}<H(\xi)^{\chi}$. For any integer $h=0, \ldots, T-1$, we have $2 q_{j+h}^{v} \leq 2 q_{j}^{v c^{T-1}}$ and, by (6.8),

$$
\begin{aligned}
\left|\xi-\frac{p_{j+h}}{q_{j+h}}\right| & \leq\left|\theta-\frac{p_{j+h}}{q_{j+h}}\right|+|\theta-\xi| \\
& \leq \frac{1}{2 q_{j+h}^{v}}+H(\xi)^{-\chi}<\frac{1}{q_{j+h}^{v}} .
\end{aligned}
$$

Thus, the inequality

$$
\left|\xi-\frac{p}{q}\right|<\frac{1}{q^{v}}
$$

has at least $T$ solutions in reduced rational numbers $p / q$ with $q \geq H(\xi)^{c_{2}(d, \varepsilon / 2)}$. By the theorem of Mignotte, we have

$$
T<c_{1}(d, \varepsilon / 2)=\exp \left\{8 \varepsilon^{-2}(\log 2 d)\right\} .
$$

Our choice for $T$ and the inequalities (6.7), (6.9) and (6.10) imply that

$$
2 q_{j}^{v c^{T}} \geq H(\xi)^{\chi} \geq q_{j}^{\chi /\left(c c_{2}(d, \varepsilon)\right)}
$$

and thus

$$
\chi \leq 2 v c^{1+c_{1}(d, \varepsilon)} c_{2}(d, \varepsilon) .
$$

We then infer from (6.11) that there are $c_{3}$ and $c_{4}$, depending only on $\varepsilon$ and $c$, such that $c_{3} \geq c$ and

$$
\chi \leq \exp \left\{c_{3} d^{c_{4}}\right\}
$$

Recalling that $w_{1}^{*}(\theta)$ does not exceed $c$, we have established the transcendence measure

$$
w_{d}^{*}(\theta) \leq \exp \left\{c_{3} d^{c_{4}}\right\}, \quad(d \geq 1),
$$

and proved that $\theta$ is not a $U$-number. Our result is slightly better than the theorem of Baker. As already noticed above, the improvement comes ultimately from a sharpening of an auxiliary lemma occurring in the proof of Roth's Theorem.

Following this approach, the use of Evertse's estimate [35] in place of the theorem of Davenport and Roth allows us [5] to show that, under the assumption of Baker's Theorem, there exists a constant $c_{5}$, depending only on $\theta$ and on $\varepsilon$, such that

$$
w_{d}^{*}(\theta) \leq(2 d)^{c_{5} \log \log 3 d}, \quad(d \geq 1) .
$$

The improvement upon (6.12) rests essentially on the improvement of Roth's lemma obtained by Evertse [34].

\section{Some recent applications of the Quantitative Subspace Theorem}

Our first result, extracted from [19], deals with the approximation to algebraic numbers by algebraic numbers. It refines the second part of the theorem of Schmidt stated in Section 3. 
Theorem (Bugeaud, 2009). Let $n$ be a positive integer. Let $\xi$ be an algebraic real number of degree greater than $n$. Then, there are a positive constant $c$, depending only on $\xi$, and infinitely many algebraic numbers $\alpha$ of degree $n$ such that

$$
|\xi-\alpha|<H(\alpha)^{-n-1+c(\log \log 3 H(\alpha))^{-1 /(2 n+6)}} .
$$

The key ingredient in the proof of this theorem is a multidimensional extension of the Cugiani-Mahler Theorem. Again, using the new result of Evertse and Ferretti [37], it is possible to replace the exponent $1 /(2 n+6)$ in $(7.1)$ by an absolute constant.

Now, we mention a few applications to the complexity of algebraic numbers, beginning with a result from [21]. Recall that the complexity function $n \mapsto p(n, \theta, b)$ has been defined in Section 4.

Theorem (Bugeaud and Evertse, 2008). Let $b \geq 2$ be an integer and $\xi$ an algebraic irrational number. Then, for any real number $\eta$ such that $\eta<1 / 11$, we have

$$
\limsup _{n \rightarrow+\infty} \frac{p(n, \xi, b)}{n(\log n)^{\eta}}=+\infty
$$

The main tools for the proof are a suitable extension of the Cugiani-Mahler Theorem and a suitable version of the Quantitative Subspace Theorem, which allows us to get an exponent of $\log n$ independent of the base $b$.

In the next statement, proved in [6], we say that a real number $\theta$ is of sublinear complexity if there exists a constant $C$ and an integer base $b \geq 2$ such that the complexity function of $\theta$ in base $b$ satisfies

$$
p(n, \theta, b) \leq C n, \quad \text { for all } n \geq 1 .
$$

Recall that a Liouville number is an irrational real number $\theta$ such that for every real number $w$, there exists a rational number $p / q$ with $|\theta-p / q|<1 / q^{w}$.

Theorem (Adamczewski and Bugeaud, 2011). Any irrational real number of sublinear complexity is either an $S$-number, or a $T$-number, or a Liouville number.

As already said, one of the main features of the theorems of Roth and Schmidt is that they are ineffective, in the sense that we cannot produce an explicit upper bound for the denominators of the solutions to (2.1) or for the height of the subspaces containing the solutions to (2.3). Consequently, the theorem of Adamczewski and Bugeaud on the complexity of algebraic numbers is ineffective, as are the weaker results from [10, 44]. It is shown in [18] that, by means of the Quantitative Subspace Theorem, it is possible to derive an explicit form of a much weaker statement.

Theorem (Bugeaud, 2008). Let $b \geq 2$ be an integer. Let $\xi$ be a real algebraic irrational number of degree $d$ and height at most $H$ with $H \geq e^{e}$. Set

$$
M=\exp \left\{10^{190}(\log (8 d))^{2}(\log \log (8 d))^{2}\right\}+2^{32 \log (240 \log (4 H))} .
$$


Then we have

$$
p(n, \xi, b) \geq\left(1+\frac{1}{M}\right) n, \quad \text { for } n \geq 1
$$

Unfortunately, the present methods do not seem to be powerful enough to get an effective version of (4.6).

We conclude by an explicit statement from [23] which confirms the conjecture of Győry, Sárközy and Stewart [48].

Theorem (Bugeaud and Luca, 2004). Let $\mathcal{A}$ be a finite set of cardinality $|\mathcal{A}|$ at least two of triples of positive integers $(a, b, c)$ with $a>b>c$. There exists a triple $(a, b, c)$ in $\mathcal{A}$ such that

$$
P[(a b+1)(a c+1)(b c+1)]>10^{-7} \log |\mathcal{A}| \log \log |\mathcal{A}| .
$$

To quantify (4.1) remains an open problem.

Acknowledgements. The author wishes to thank Jan-Hendrik Evertse and Kálmán Györy for their remarks on a preliminary version of this text.

\section{References}

[1] B. Adamczewski and Y. Bugeaud, On the complexity of algebraic numbers, II. Continued fractions, Acta Math. 195 (2005), 1-20.

[2] B. Adamczewski and Y. Bugeaud, On the complexity of algebraic numbers I. Expansions in integer bases, Ann. of Math. 165 (2007), 547-565.

[3] B. Adamczewski and Y. Bugeaud, On the Maillet-Baker continued fractions, J. reine angew. Math. 606 (2007), 105-121.

[4] B. Adamczewski and Y. Bugeaud, Palindromic continued fractions, Ann. Inst. Fourier (Grenoble) 57 (2007), 1557-1574.

[5] B. Adamczewski et Y. Bugeaud, Mesures de transcendance et aspects quantitatifs de la méthode de Thue-Siegel-Roth-Schmidt, Proc. London Math. Soc. 101 (2010), $1-31$.

[6] B. Adamczewski et Y. Bugeaud, Nombres réels de complexité sous-linéaire : mesures d'irrationalité et de transcendance, J. reine angew. Math. À paraître.

[7] B. Adamczewski, Y. Bugeaud, and L. Davison, Continued fractions and transcendental numbers, Ann. Inst. Fourier (Grenoble) 56 (2006), 2093-2113.

[8] B. Adamczewski, Y. Bugeaud et F. Luca, Sur la complexité des nombres algébriques, C. R. Acad. Sci. Paris 339 (2004), 11-14. 
[9] P. B. Allen, On the multiplicity of linear recurrence sequences, J. Number Theory 126 (2007), 212-216.

[10] J.-P. Allouche, Nouveaux résultats de transcendance de réels à développements non aléatoire, Gaz. Math. 84 (2000), 19-34.

[11] F. Amoroso and E. Viada, Small points on subvarieties of a torus, Duke Math. J. 150 (2009), 407-442.

[12] F. Amoroso and E. Viada, On the zeros of linear recurrence sequences. Preprint.

[13] A. Baker, On Mahler's classification of transcendental numbers, Acta Math. 111 (1964), 97-120.

[14] Yu. Bilu, The many faces of the subspace theorem [after Adamczewski, Bugeaud, Corvaja, Zannier...]. Séminaire Bourbaki. Vol. 2006/2007. Astérisque No. 317 (2008), Exp. No. 967, vii, 1-38.

[15] E. Bombieri and W. Gubler, Heights in Diophantine geometry. New Mathematical Monographs, vol. 4, Cambridge University Press, 2006.

[16] Y. Bugeaud, Approximation by algebraic numbers, Cambridge Tracts in Mathematics 160, Cambridge, 2004.

[17] Y. Bugeaud, Extensions of the Cugiani-Mahler Theorem, Ann. Scuola Normale Superiore di Pisa 6 (2007), 477-498.

[18] Y. Bugeaud, An explicit lower bound for the block complexity of an algebraic number, Atti Accad. Naz. Lincei Cl. Sci. Fis. Mat. Natur. Rend. Lincei (9) Mat. Appl. 19 (2008), 229-235.

[19] Y. Bugeaud, On the approximation to algebraic numbers by algebraic numbers, Glas. Mat. 44 (2009), 323-331.

[20] Y. Bugeaud, P. Corvaja, and U. Zannier, An upper bound for the G.C.D. of $a^{n}-1$ and $b^{n}-1$, Math. Z. 243 (2003), 79-84.

[21] Y. Bugeaud and J.-H. Evertse, On two notions of complexity of algebraic numbers, Acta Arith. 133 (2008), 221-250.

[22] Y. Bugeaud and J.-H. Evertse, Approximation of complex algebraic numbers by algebraic numbers of bounded degree, Ann. Scuola Normale Superiore di Pisa 8 (2009), $333-368$.

[23] Y. Bugeaud and F. Luca, A quantitative lower bound for the greatest prime factor of $(a b+1)(b c+1)(c a+1)$, Acta Arith. 114 (2004), 275-294.

[24] P. Bundschuh und A. Pethő, Zur Transzendenz gewisser Reihen, Monatsh. Math. 104 (1987), 199-223.

[25] P. Corvaja and U. Zannier, Diophantine equations with power sums and universal Hilbert sets, Indag. Math. (N.S.) 9 (1998), 317-332. 
[26] P. Corvaja and U. Zannier, Some new applications of the subspace theorem, Compositio Math. 131 (2002), 319-340.

[27] P. Corvaja and U. Zannier, On the greatest prime factor of $(a b+1)(a c+1)$, Proc. Amer. Math. Soc. 131 (2003), 1705-1709.

[28] M. Cugiani, Sull'approssimazione di numeri algebrici mediante razionali, Collectanea Mathematica, Pubblicazioni dell'Istituto di matematica dell'Università di Milano 169, Ed. C. Tanburini, Milano, pagg. 5 (1958).

[29] M. Cugiani, Sulla approssimabilità dei numeri algebrici mediante numeri razionali, Ann. Mat. Pura Appl. 48 (1959), 135-145.

[30] M. Cugiani, Sull'approssimabilità di un numero algebrico mediante numeri algebrici di un corpo assegnato, Boll. Un. Mat. Ital. 14 (1959), 151-162.

[31] H. Davenport and K. F. Roth, Rational approximations to algebraic numbers, Mathematika 2 (1955), 160-167.

[32] E. Dubois et G. Rhin, Approximations rationnelles simultanées de nombres algébriques réels et de nombres algébriques p-adiques. In: Journées Arithmétiques de Bordeaux (Conf., Univ. Bordeaux, 1974), pp. 211-227. Astérisque, Nos. 24-25, Soc. Math. France, Paris, 1975.

[33] J.-H. Evertse, On sums of $S$-units and linear recurrences, Compositio Math. 53 (1984), 225-244.

[34] J.-H. Evertse, An explicit version of Faltings' product theorem and an improvement of Roth's lemma, Acta Arith. 73 (1995), 215-248.

[35] J.-H. Evertse, The number of algebraic numbers of given degree approximating a given algebraic number. In: Analytic number theory (Kyoto, 1996), 53-83, London Math. Soc. Lecture Note Ser. 247, Cambridge Univ. Press, Cambridge, 1997.

[36] J.-H. Evertse, On the Quantitative Subspace Theorem, Zapiski Nauchnyk Seminarov POMI 377 (2010), 217-240.

[37] J.-H. Evertse and R. G. Ferretti, A further quantitative improvement of the Absolute Subspace Theorem. Preprint.

[38] J.-H. Evertse and K. Györy, Finiteness criteria for decomposable form equations, Acta Arith. 50 (1988), 357-379.

[39] J.-H. Evertse and K. Györy, The number of families of solutions of decomposable form equations, Acta Arith. 80 (1997), 367-394.

[40] J.-H. Evertse, K. Győry, C. L. Stewart, and R. Tijdeman, On S-unit equations in two unknowns, Invent. Math. 92 (1988), 461-477.

[41] J.-H. Evertse, K. Győry, C. L. Stewart, and R. Tijdeman, S-unit equations and their applications. In: New advances in transcendence theory (Durham, 1986), 110-174, Cambridge Univ. Press, Cambridge, 1988. 
[42] J.-H. Evertse and H.P. Schlickewei, A quantitative version of the Absolute Subspace Theorem, J. reine angew. Math. 548 (2002), 21-127.

[43] J.-H. Evertse, H.P. Schlickewei, and W. M. Schmidt, Linear equations in variables which lie in a multiplicative group, Ann. of Math. 155 (2002), 807-836.

[44] S. Ferenczi and Ch. Mauduit, Transcendence of numbers with a low complexity expansion, J. Number Theory 67 (1997), 146-161.

[45] K. Győry, Some recent applications of S-unit equations. Journées Arithmétiques, 1991 (Geneva). Astérisque No. 209 (1992), 11, 17-38.

[46] K. Györy, On the numbers of families of solutions of systems of decomposable form equations, Publ. Math. Debrecen 42 (1993), 65-101.

[47] K. Györy, On the irreducibility of neighbouring polynomials, Acta Arith. 67 (1994), $283-294$.

[48] K. Györy, A. Sárközy and C. L. Stewart, On the number of prime factors of integers of the form $a b+1$, Acta Arith. 74 (1996), 365-385.

[49] S. Hernández and F. Luca, On the largest prime factor of $(a b+1)(a c+1)(b c+1)$, Bol. Soc. Mat. Mexicana 9 (2003), 235-244.

[50] J. F. Koksma, Über die Mahlersche Klasseneinteilung der transzendenten Zahlen und die Approximation komplexer Zahlen durch algebraische Zahlen, Monatsh. Math. Phys. 48 (1939), 176-189.

[51] M. Laurent, Équations diophantiennes exponentielles, Invent. Math. 78 (1984), 299327.

[52] K. Mahler, Zur Approximation der Exponentialfunktionen und des Logarithmus. I, II, J. reine angew. Math. 166 (1932), 118-150.

[53] K. Mahler, Lectures on Diophantine approximation, Part 1: $g$-adic numbers and Roth's theorem, University of Notre Dame, Ann Arbor, 1961.

[54] K. Mahler, Some suggestions for further research, Bull. Austral. Math. Soc. 29 (1984), 101-108.

[55] M. Mignotte, Quelques remarques sur l'approximation rationnelle des nombres algébriques, J. reine angew. Math. 268/269 (1974), 341-347.

[56] M. Mignotte, An application of W. Schmidt's theorem: transcendental numbers and golden number, Fibonacci Quart. 15 (1977), 15-16.

[57] A. J. van der Poorten and H. P. Schlickewei, The growth condition for recurrence sequences. Macquarie Univ. Math. Rep. 82-0041, North Ryde, Australia (1982).

[58] D. Ridout, Rational approximations to algebraic numbers, Mathematika 4 (1957), 125-131.

[59] K. F. Roth, Rational approximations to algebraic numbers, Mathematika 2 (1955), 1-20; corrigendum, 168. 
[60] H. P. Schlickewei, Die p-adische Verallgemeinerung des Satzes von Thue-Siegel-RothSchmidt, J. reine angew. Math. 288 (1976), 86-105.

[61] H. P. Schlickewei, Linearformen mit algebraischen koeffizienten, Manuscripta Math. 18 (1976), 147-185.

[62] H. P. Schlickewei, The p-adic Thue-Siegel-Roth-Schmidt theorem, Arch. Math. (Basel) 29 (1977), 267-270.

[63] W. M. Schmidt, Über simultane Approximation algebraischer Zahlen durch Rationale, Acta Math. 114 (1965) 159-206.

[64] W. M. Schmidt, On simultaneous approximations of two algebraic numbers by rationals, Acta Math. 119 (1967), 27-50.

[65] W. M. Schmidt, Simultaneous approximations to algebraic numbers by rationals, Acta Math. 125 (1970), 189-201.

[66] W. M. Schmidt, Norm form equations, Ann. of Math. 96 (1972), 526-551.

[67] W. M. Schmidt, Diophantine Approximation. Lecture Notes in Mathematics 785, Springer, 1980.

[68] W. M. Schmidt, The subspace theorem in Diophantine approximation, Compositio Math. 69 (1989), 121-173.

[69] W. M. Schmidt, The number of solutions of norm form equations, Trans. Amer. Math. Soc. 317 (1990), 197-227.

[70] W. M. Schmidt, Diophantine approximations and Diophantine equations. Lecture Notes in Mathematics 1467, Springer, 1991.

[71] W. M. Schmidt, Zeros of linear recurrence sequences, Publ. Math. Debrecen 56 (2000), 609-630.

[72] Th. Schneider, Über die Approximation algebraischer Zahlen, J. reine angew. Math. 175 (1936), 182-192.

[73] G. Troi and U. Zannier, Note on the density constant in the distribution of selfnumbers. II, Boll. Unione Mat. Ital. Sez. B Artic. Ric. Mat. (8) 2 (1999), 397-399.

[74] U. Zannier, Some applications of diophantine approximation to diophantine equations (with special emphasis on the Schmidt subspace theorem). Forum, Udine, 2003.

Yann Bugeaud

Université de Strasbourg

Mathématiques

7, rue René Descartes

67084 STRASBOURG Cedex (FRANCE)

bugeaud@math.unistra.fr 